\title{
Adaptation to Resistance Training Is Associated with Higher Phagocytic (but Not Oxidative) Activity in Neutrophils of Older Women
}

\author{
João Bartholomeu-Neto, ${ }^{1}$ Ciro José Brito, ${ }^{2}$ Otávio Toledo Nóbrega, ${ }^{3}$ \\ Vinícius Carolino Sousa, ${ }^{3}$ Juliana Oliveira Toledo, ${ }^{3}$ Roberta Silva Paula, ${ }^{3}$ \\ David Junger Fonseca Alves, ${ }^{1}$ Aparecido Pimentel Ferreira, ${ }^{4}$ \\ Clayton Franco Moraes, ${ }^{1,3}$ and Cláudio Córdova ${ }^{1}$ \\ ${ }^{1}$ Catholic University of Brasília, 71966-700 Brasília, DF, Brazil \\ ${ }^{2}$ Federal University of Juiz de Fora, 36036-330 Governador Valadares, MG, Brazil \\ ${ }^{3}$ University of Brasília, 70910-900 Brasília, DF, Brazil \\ ${ }^{4}$ Paulista University, 70390-130 Brasília, DF, Brazil
}

Correspondence should be addressed to Otávio Toledo Nóbrega; otnobrega@gmail.com

Received 10 June 2015; Revised 28 August 2015; Accepted 10 September 2015

Academic Editor: Eileen Uribe-Querol

Copyright (C) 2015 João Bartholomeu-Neto et al. This is an open access article distributed under the Creative Commons Attribution License, which permits unrestricted use, distribution, and reproduction in any medium, provided the original work is properly cited.

Failure in antimicrobial activity contributes to high morbidity and mortality in the geriatric population. Little is known about the potential effect of resistance training (RT) on the functional properties of the innate immunity. This study aimed to investigate the influence of long-term RT on the endocytic and oxidative activities of neutrophils and monocytes in healthy older women. Our results indicate that the phagocytosis index (PhI) of neutrophils (but not of monocytes) in the RT-adapted group was significantly higher $(P<0.001$; effect size, $(d)=0.90,95 \% \mathrm{CI}$ : [0.75-1.04]) compared to that in sedentary subjects. In contrast, the oxidative activity of either neutrophils or monocytes was not significantly influenced by RT. Also, total energy and carbohydrate intake as well as serum IL6 levels had a significant influence on the phagocytic activity of neutrophils $(P=0.04)$, being considered in the model. Multivariate regression identified the physical condition of the subject $(\beta=0.425 ; P=0.01)$ as a significant predictor of PhI. In conclusion, circulating neutrophils of older women adapted to a long-term RT program expressed higher phagocytic activity.

\section{Introduction}

The cascade of biological events that makes up the innate defense against infectious agents is a vital part of the immune system. Typically, this process is characterized by acute response triggered by the rapid increase in circulating inflammatory mediators [1]. However, during immunosenescence, there is complex remodeling of the immune system, characterized by exacerbation of the basal, possibly nonimmunologically derived profile of proinflammatory mediators and of reactive oxygen species, a phenomenon known as inflammaging [2]. This scenario is more evident after the fourth decade of life, when increased susceptibility to cancer, infections, and metabolic disorders is observed $[3,4]$.
Although the effects of aging on the innate immune system are not fully elucidated [5-7], it is assumed that this senescence-associated, subclinical inflammatory, and oxidative processes constitute a compensatory mechanism due to the decline of the endocytic capacity and reduced production of superoxides by phagocytic elements, among other agetriggered flaws of the immune function. Failure in neutrophil properties has been postulated as an important predictor of morbidity and mortality in the geriatric population [5].

Further studies aimed at exploring nonpharmacological interventions with potential to reverse the detrimental aspects of immunosenescence should be carried out $[8,9]$. Although results already in the literature disclose benefits of resistance training (RT) on the systemic proinflammatory 


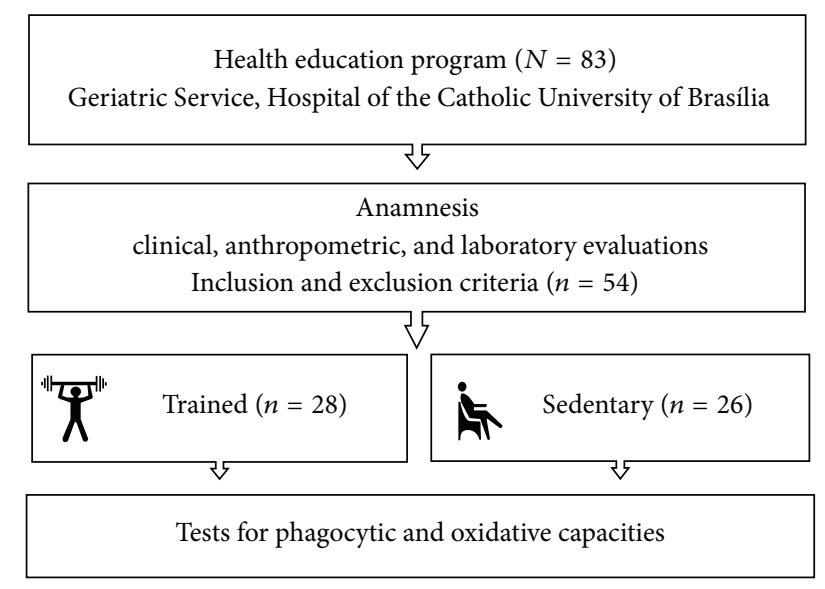

Figure 1: Procedures for sample selection and data collection.

milieu of elderly subjects [10-12], little is known about the influence of RT on the functional response of cells that form the first line of defense of the immune system, particularly in this age stratum. This study aimed to investigate the influence of long-term RT on the phagocytic and oxidative activities of neutrophils and monocytes in apparently healthy older women. The intake of the main macronutrients and the circulating levels of important serum mediators of immunosenescence and endocrinosenescence were taken as potential confounding factors and considered herein. Our hypothesis is that chronic physiological adaptations induced by RT can modulate the endocytic and oxidative capacities of peripheral phagocytes in older women.

\section{Materials and Methods}

2.1. Participants. The participants of this study were elderly women living in the community regularly followed by health education programs for prevention of chronic disorders developed by the Geriatric Service of the Catholic University of Brasilia Hospital, Brazil, known as the Prognosis and Therapeutics in Geriatrics (ProTeGer) study [13, 14]. After inspection of medical records, patients were excluded due to the following: uncontrolled type 2 diabetes, obesity, having smoked > 100 cigarettes over a lifetime, and/or having regularly consumed one dose ( $12 \mathrm{~g})$ of alcohol per week or more over the last 12 months. Other exclusion criteria were use of immunomodulatory drugs or presence of neoplastic processes, acute infectious, and/or inflammatory signs at the time of clinical evaluations. Figure 1 presents the chart of procedures adopted during this study.

The Lipschitz equation was used to determine the body mass index (BMI) [15]. The waist-to-hip ratio (WHR) was calculated based on waist circumference measurements at the midpoint between the last rib and the iliac crest and hip circumference in the greater trochanter. Body mass was measured with participants barefoot and wearing light clothing on a calibrated scale with $100 \mathrm{~g}$ accuracy (Filizola, São Paulo, SP, Brazil). Height was measured with stadiometer with accuracy of $1 \mathrm{~cm}$. Body composition measurements were performed at the Laboratory of Image of the Institution using dual energy X-ray absorptiometry (DXA; Lunar DPXIQ model, software version 4.7e, Lunar Radiation Corp., Madison, WI, USA), with individuals in the supine position on a horizontal platform, with slightly apart and relaxed legs, arms at body sides, and palms down. The software provided fat and fat-free masses. In addition to absolute masses, relative fat and fat-free measures were obtained with adjustment to height $\left(\mathrm{kg} / \mathrm{m}^{2}\right)$. The equipment was calibrated as recommended by the manufacturer. All examinations were performed by the same trained researcher.

2.2. Nutritional Assessment. Dietary data were determined based on food records of 3 days ( 2 working days and 1 day in the weekend), which is as accurate as records of 4 or 7 days $[16,17]$. The food record was completed at home with patients being instructed to record food consumption in terms of number and size of portions. Values for each individual patient were expressed as the average intake of the 3 days reported. To ensure completion of food records, the staff of nutritionists provided personal or telephone assistance. The forms were returned during a clinical interview in which the amounts and types of foods were reviewed. Dietary contents were calculated using the Diet-Pro software, version 4.0 (A.S. Sistemas, Viçosa, MG, Brazil), configured for international food tables and complemented with a table for local food products [18]. Dietary intake of total protein, carbohydrates, and lipids was expressed as percentage of total energy and included in the analysis as continuous variables.

\subsection{Resistance Training Prescription. For the RT prescription,} the 1-maximum-repetition (MR) percentage was obtained according to methodology described by Kraemer et al. [19]. The group under RT was composed of volunteer women who have attended a moderate-intensity physical training (70\% of $1 \mathrm{MR}$ ) consisting of exercise sessions three times a week on alternate days for $8.6 \pm 0.3$ months, with three sets of 12 repetitions per exercise at moderate speed and 1 minute of rest between sets. Each 50-minute session included nine exercises: horizontal leg press, knee extension, knee flexion, bench press, triceps extension in the pulley, biceps curling, seated rowing, plantar flexion, and abdominals. Whenever one participant was able to perform 13 repetitions or more in the third series of a given movement in the last weekday of training, loads were increased [20]. All equipment was manufactured by Righetto (Campinas, SP, Brazil), and warmup exercises for 10 min preceded each training session.

All subjects were sedentary at baseline. The control group consisted of women who remained sedentary and participated during nine months in occupational activities not related to physical activity offered by the health education program. Attendance to either physical or occupational activities was entirely voluntary and freely chosen by each olderwoman, as long as the three-session-per-week regimen was fulfilled when inscribed in RT-training. The study protocol was approved by the Research Ethics Committee of the institution and conducted in accordance with the Declaration of Helsinki. 
2.4. Total and Differential Count of Leukocytes. Blood samples were diluted at 1:20 in Turk's solution. Leukocytes were counted in a Neubauer chamber (Labex, Aparecida de Goiânia, GO, Brazil) with concentration $\left(\right.$ cells $/ \mathrm{mm}^{3}$ ) determined under optical microscopy by the equation $[((Q 1+$ Q2 + Q3 + Q4)/4) $\times 200$ ], with $Q n$ as the number of cells in the quadrant $n$ of the chamber and 200 as the product of 10 (conversion factor to $1 \mathrm{~mm}^{3}$, count area depth) and 20 (dilution factor). For differential leukocyte count, 100 cells in randomly distributed fields were considered.

2.5. Analysis of Serum Mediators. For measurement of immune mediators, blood samples were collected in the morning (8:00 to 9:30 a.m.) in tubes without endotoxin. For RT-adapted individuals, samples were collected at the end of the intervention period, along with samples from control subjects. Serum was separated within 1 hour after collection and stored at $-80^{\circ} \mathrm{C}$ until processing day. Samples were analyzed using the enzyme-linked immunosorbent assay method (ELISA) with kits specific for interleukin 6 (IL6) and for tumor necrosis factor-alpha (TNF $\alpha)$ (eBioscience, San Diego, CA). Serum IGF1 levels were determined using automated Immulite 2000 Siemens system (Los Angeles, CA, USA). All samples were analyzed in duplicate.

2.6. Phagocytic Capacity Test. The endocytic capacity of circulating monocytes and neutrophils was tested in vitro by a Saccharomyces cerevisiae phagocytosis assay adapted from Muniz-Junqueira et al. [21]. Briefly, blood samples $(40 \mu \mathrm{L})$ were added in duplicate onto glass slides with $7 \mathrm{~mm}$ wide excavations and incubated in humid chamber for $45 \mathrm{~min}$ at $37^{\circ} \mathrm{C}$. Then, slides were washed with PBS solution, $\mathrm{pH}$ 7.2 at $37^{\circ} \mathrm{C}$. Adherent cells were incubated with $2.5 \times 10^{5}$ yeasts in $20 \mu \mathrm{L}$ of Hanks-Tris solution (Sigma, St. Louis, MO, USA), pH 7.2, with 10\% fetal bovine serum (FBS) (Cultilab, Campinas, Brazil). After $30 \mathrm{~min}$ of incubation in humid chamber at $37^{\circ} \mathrm{C}$, slides were rinsed with $\mathrm{PBS}$ solution at $37^{\circ} \mathrm{C}$, washed with Hanks-Tris solution with $30 \%$ FBS at $37^{\circ} \mathrm{C}$, fixed with methanol, and stained with Giemsa solution. The number of S. cerevisiae phagocytosed by 200 monocytes or 200 neutrophils in individual preparations was evaluated by optical microscopy. The fields used for counting were randomly selected and all monocytes or neutrophils found were examined. The phagocytosis index (PhI) was calculated as the product of the following factors: (a) average number of S. cerevisiae phagocytosed by neutrophils or monocytes and (b) proportion of neutrophils or monocytes involved in the phagocytosis.

Yeast suspension was prepared following the method described by Lachmann and Hobart [22]. Briefly, $50 \mathrm{~g}$ tablet of fresh yeast (Fleischmann) was dissolved in $220 \mathrm{~mL}$ of a $0.05 \mathrm{M}$ isotonic, phosphate buffered saline solution (PBS), $\mathrm{pH} 7.2$, autoclaved at $120^{\circ} \mathrm{C}$ for 30 minutes, and then washed for 3 times in PBS by centrifugation at 4,000 rpm for $5 \mathrm{~min}$. This procedure was repeated to obtain a clear supernatant. The sediment was resuspended in $28 \mathrm{~mL}$ of PBS containing 2-mercaptoethanol at $0.1 \mathrm{M}$. After two hours of stirring at $37^{\circ} \mathrm{C}$, the suspension was washed again and the sediment was resuspended in $55 \mathrm{~mL}$ in PBS solution containing $0.02 \mathrm{M}$ iodoacetamide. After further stirring at $37^{\circ} \mathrm{C}$ for two hours, the suspension was washed three times, resuspended in $220 \mathrm{~mL}$ of PBS, autoclaved once more, and washed with PBS by centrifugation to obtain a clear supernatant, being finally resuspended in $110 \mathrm{~mL}$ of Veronal buffer of $\mathrm{pH} 7.2$ containing sodium azide $(200 \mathrm{mg} / \mathrm{L})$. The yeast suspension was stored at $4^{\circ} \mathrm{C}$ for use for a period not exceeding one week after its production. Prior to each assay, the yeast suspension was washed in PBS, suspended in equal volume of Hanks-Tris solution, quantified, and used immediately.

2.7. Oxidative Capacity Test. The oxidative capacity test was adapted from technique described by Park et al. [23] to evaluate the production of reactive oxygen species based on the principle of reduction of nitroblue tetrazolium (NBT) up to its insoluble form (formazan). Briefly, phagocytes adhered to excavation, as previously described, were incubated with Hanks-Tris solution containing $0.05 \%$ of NBT for $20 \mathrm{~min}$ at $37^{\circ} \mathrm{C}$ in humid chamber. The slides were rinsed, fixed with methanol, and stained with aqueous solution containing $1.4 \%$ safranin and $28.6 \%$ glycerol. The proportion of phagocytes that reduced NBT in their cytoplasm was determined by counting 200 neutrophils and 200 monocytes found. Positive, NBT-reducing phagocytes were those that presented the typical blackish-blue cytoplasmic precipitate that is compatible with formazan formation.

The phagocytic and oxidative capacities were intended to be assessed simultaneously and for 2 or 3 subjects at once. After assaying all subjects, our first-round success rate scored at $90 \%$, with minimum slide quality achieved whenever distinction of leucocytes and identification of yeasts were possible by visual inspection. Blanks were filled by new invitations for blood draw until data was completed for the whole sample. All tests were performed by the same trained biologist.

2.8. Statistical Analysis. Data analysis was performed using the Statistical Package for Social Sciences (SPSS) for Windows (version 8.0). The Kolmogorov-Smirnov test was used to test the data normality. To obtain a close-to-normal distribution, data were logarithmically transformed $\left(\log _{10}\right)$, whenever necessary. Student's $t$-test for independent samples was used to compare groups in terms of body composition, calorie intake, age, white blood cell count, and levels of proinflammatory mediators. Analysis of covariance (ANCOVA) was performed using both groups (RT versus sedentary) as independent variables to investigate possible differences induced by exercise on the phagocytic activity, with IL6, BMI, and caloric intake measures as covariant. Associations of body composition, total protein, carbohydrates, lipids, IL6, TNF $\alpha$, and IGF1 measurements with phagocytosis of monocytes and neutrophils were analyzed using Pearson's product-moment correlation coefficient. Where appropriate, the effect size $(d)$ and confidence interval (95\% CI $=d \pm Z_{0.025} S_{d}$ ) were estimated. Multivariate linear regression with a stepwise variable selection was used in order to test the relationship between phagocytic activity and potential predictors of the regression model. Assumptions of normality, homogeneity, and independence of wastes were investigated, the former two by visual inspection and the latter by the Durbin-Watson statistics $(d=1.7)$. 
TABLE 1: Characteristics of the sample.

\begin{tabular}{|c|c|c|c|}
\hline & $\begin{array}{l}\text { Sedentary } \\
(n=26)\end{array}$ & $\begin{array}{l}\text { Trained } \\
(n=28)\end{array}$ & $P^{*}$ \\
\hline Age, years & $72.0 \pm 6.9$ & $70.6 \pm 5.7$ & 0.406 \\
\hline BMI, $\mathrm{kg} / \mathrm{m}^{2}$ & $27.9 \pm 4.4$ & $29.5 \pm 5.5$ & 0.223 \\
\hline Total fat-free mass, kg & $36.5 \pm 2.4$ & $39.1 \pm 5.9$ & 0.040 \\
\hline $\begin{array}{l}\text { Relative fat-free mass, } \\
\mathrm{kg} / \text { height }^{2}\end{array}$ & $15.8 \pm 0.9$ & $16.4 \pm 1.8$ & 0.090 \\
\hline Total fat mass, $\mathrm{kg}$ & $24.9 \pm 7.2$ & $25.8 \pm 8.1$ & 0.121 \\
\hline Relative fat mass, $\mathrm{kg} / \mathrm{height}^{2}$ & $11.8 \pm 3.1$ & $12.9 \pm 3.8$ & 0.208 \\
\hline Waist circumference, $\mathrm{cm}$ & $97.4 \pm 9.2$ & $99.8 \pm 12.2$ & 0.406 \\
\hline Calorie intake, $10^{3} \mathrm{kcal}^{*}$ & $2.2 \pm 0.6$ & $1.7 \pm 0.5$ & 0.001 \\
\hline Total carbohydrate intake, $\%$ & $50.1 \pm 4.9$ & $47.4 \pm 8.3$ & 0.050 \\
\hline Total lipid intake, $\%$ & $34.1 \pm 4.9$ & $36.0 \pm 7.6$ & 0.129 \\
\hline Total protein intake, $\%$ & $14.7 \pm 3.8$ & $15.5 \pm 3.5$ & 0.424 \\
\hline $\begin{array}{l}\text { Systolic blood pressure, } \\
\mathrm{mmHg}\end{array}$ & $134.3 \pm 13.0$ & $125.8 \pm 14.1$ & 0.022 \\
\hline $\begin{array}{l}\text { Diastolic blood pressure, } \\
\mathrm{mm} \mathrm{Hg}\end{array}$ & $81.9 \pm 9.9$ & $80.8 \pm 9.5$ & 0.670 \\
\hline IGF1, ng/mL & $0.60 \pm 0.17$ & $0.56 \pm 0.19$ & 0.410 \\
\hline Leukocyte numbers, $\mathrm{mm}^{3}$ & $5.2 \pm 2.1$ & $5.7 \pm 2.2$ & 0.401 \\
\hline Neutrophils, \% & $55.4 \pm 11.7$ & $53.2 \pm 9.7$ & 0.449 \\
\hline Segmented & $53.4 \pm 11.3$ & $52.1 \pm 9.5$ & - \\
\hline Banded & $2.0 \pm 1.5$ & $1.1 \pm 1.4$ & - \\
\hline Monocytes, \% & $3.9 \pm 2.2$ & $3.8 \pm 2.8$ & 0.910 \\
\hline Lymphocytes, \% & $37.9 \pm 10.4$ & $39.8 \pm 10.1$ & 0.494 \\
\hline Eosinophils, \% & $2.7 \pm 2.6$ & $3.1 \pm 2.7$ & 0.608 \\
\hline $\mathrm{TNF} \alpha \mathrm{pg} / \mathrm{mL}$ & $0.73 \pm 0.29$ & $0.57 \pm 0.20$ & 0.036 \\
\hline IL6 pg/mL L $^{*}$ & $0.63 \pm 0.12$ & $0.44 \pm 0.36$ & 0.002 \\
\hline
\end{tabular}

Values are expressed as mean \pm standard deviation. ${ }^{*}$ Statistical difference $P<0.050$.

Variance inflation factor was used for the diagnosis of possible collinearity among variables (VIF's $<1.3$ ). $P<0.05$ was considered statistically significant in bilateral tests.

\section{Results}

After applying the exclusion criteria, 54 older women (71.3 \pm 6.3 years) composed two groups, 28 in the RT-adapted subset and 26 as a sedentary counterpart. Participants' characteristics are described in Table 1 . When compared to the sedentary group, women adapted to RT exhibited reduced average values for total caloric intake $(\sim 23 \%)$ and systolic blood pressure $(\sim 6 \%)$. Similarly, reduced circulating levels of proinflammatory biomarkers $\log _{10}$ IL6 $(\sim 30 \%)$ and $\log _{10}$ $\mathrm{TNF} \alpha(\sim 22 \%)$ were observed in the RT-adapted group.

Concerning phagocytosis by monocytes and neutrophils, comparisons between groups are shown in Table 2. Increased phagocytic capacity of neutrophils was observed among RTadapted participants compared to the sedentary group, represented by both greater proportion of $S$. cerevisiae captured $(\sim 31 \% ; P<0.001)$ and a higher frequency of phagocyting
TABLE 2: Phagocytic and oxidative activities in the samples.

\begin{tabular}{lccc}
\hline & $\begin{array}{c}\text { Sedentary } \\
(n=26)\end{array}$ & $\begin{array}{c}\text { Trained } \\
(n=28)\end{array}$ & $P^{*}$ \\
\hline $\begin{array}{l}\text { S. cerevisiae } \\
\text { phagocyte/neutrophils, } n\end{array}$ & $2.2 \pm 0.5$ & $2.9 \pm 0.9$ & $<0.001$ \\
$\begin{array}{l}\text { S. cerevisiae } \\
\text { phagocyte/monocytes, } n\end{array}$ & $1.4 \pm 0.2$ & $1.5 \pm 0.3$ & 0.416 \\
$\begin{array}{l}\text { Neutrophils in phagocytosis, \% } \\
\text { Monocytes in phagocytosis, \% }\end{array}$ & $60.5 \pm 19.6$ & $71.2 \pm 19.1$ & 0.033 \\
$\begin{array}{l}\text { Index of neutrophils } \\
\text { phagocytosis }\end{array}$ & $140.4 \pm 69.5$ & $221.2 \pm 105.8$ & $<0.001$ \\
$\begin{array}{l}\text { Index of monocytes } \\
\text { phagocytosis }\end{array}$ & $53.8 \pm 22.4$ & $60.5 \pm 18.2$ & 0.160 \\
$\begin{array}{l}\text { Neutrophils reduced NBT, \% } \\
\text { Monocytes reduced NBT, \% }\end{array}$ & $51.3 \pm 23.0$ & $45.3 \pm 26.4$ & 0.328 \\
\hline
\end{tabular}

Values are expressed as mean \pm standard deviation. ANCOVA adjusted for IL6, BMI, and calorie intake. ${ }^{*}$ Statistical difference set at $P<0.05$.

cells ( $18 \%, P=0.033)$. Thus, the PhI of neutrophils was significantly higher in the RT-adapted group $(\sim 57 \% ; P<0.001$, $d=0.90,95 \%$ CI: [0.75-1.04]) compared to the group without exercise. Among covariates, total energy intake and the intake of carbohydrates had a significant influence on the phagocytic activity of neutrophils $(F(1.53)=3.67 ; P=0.04)$, being necessarily considered to be covariables in our models. In contrast, no statistically significant differences in the phagocytic ability of monocytes between groups were observed. The production of reactive oxygen species in neutrophils and monocytes, as assessed by the proportion of cells that promoted NBT reduction, was not significantly influenced by RT.

Results of correlation tests with inclusion of all participants showed a significant negative association of the neutrophils' PhI with circulating levels of IL6 $(r=-0.29 ; P=0.03)$ and the intake of total carbohydrates $(r=-0.32 ; P=0.01)$ (Figure 2). There were no significant associations between $\mathrm{PhI}$ and circulating levels of IGF1 and TNF $\alpha$. Multivariate regression analysis identified the physical condition of subjects (RT-adapted or sedentary) as the most significant variable to predict PhI $(\beta=0.425 ; P=0.01)$. $\log _{10}$ IL6 and caloric intake were excluded from the model.

\section{Discussion}

In the field of immunology, it is generally recognized that rapid activation and recruitment of neutrophils reflect the integrity of important signaling pathways involved with the initiation of immune responses, to result in protective inflammation [24, 25]. The main findings of this study are consistent with a higher phagocytic activity of circulating neutrophils in older women adapted to a long-term RT program in relation to the sedentary group. Since the disparity in $\mathrm{PhI}$ between groups was not mitigated with statistical control for potentially confounding factors (as nutritional profile, anthropometry, and cytokine levels), it is suggested that the benefits of RT may be the result of independent physiological mechanisms (e.g., hormonal), as advocated by evidence presented elsewhere [26]. 


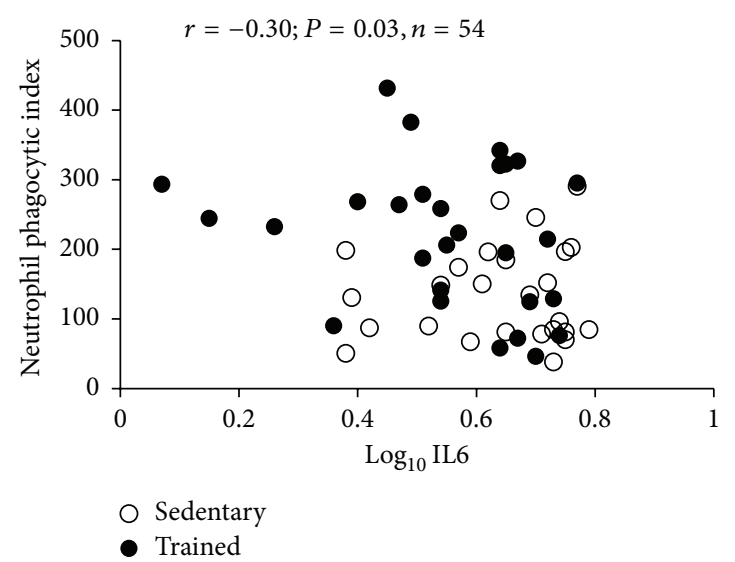

(a)

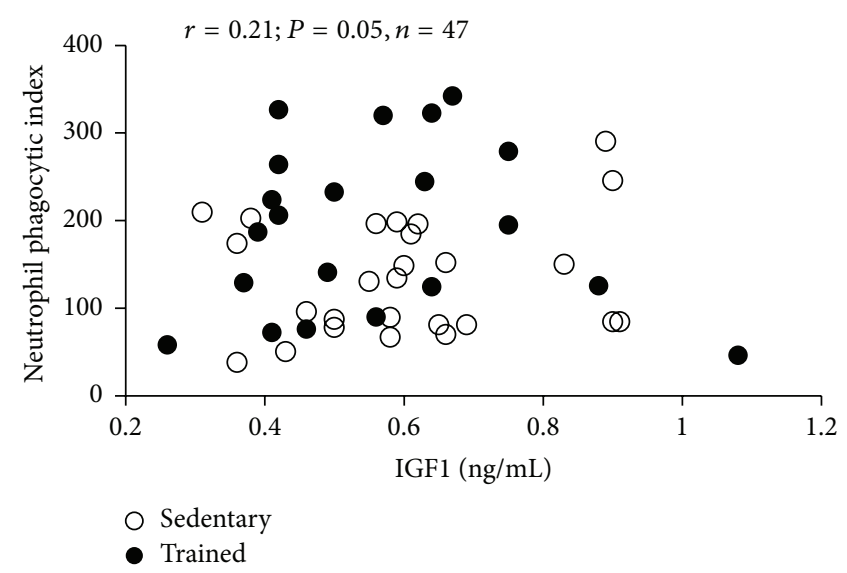

(c)

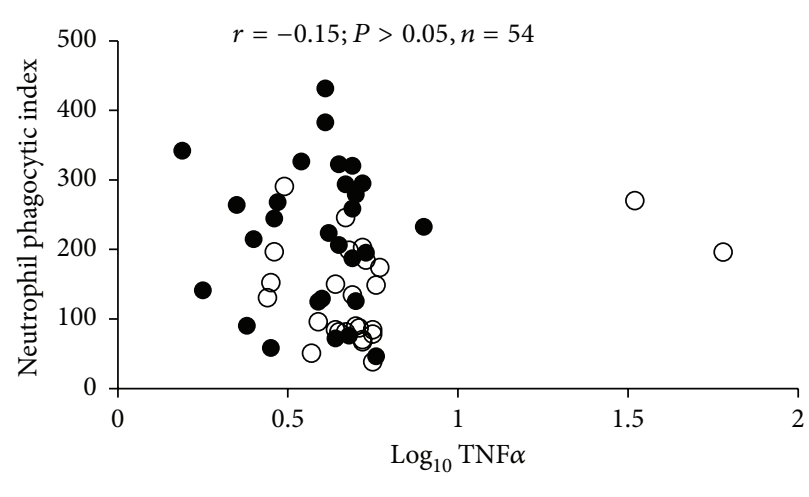

O Sedentary

- Trained

(b)

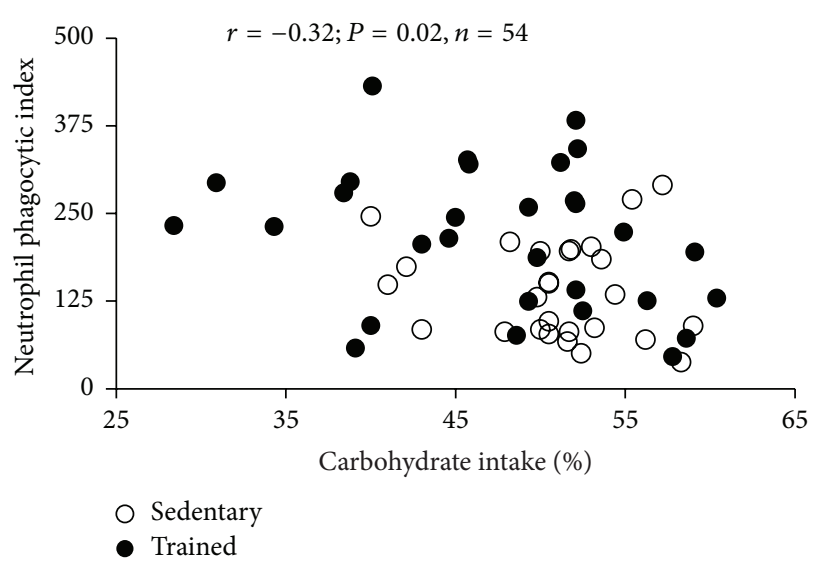

(d)

FIGURE 2: Correlations of $\log _{10}$ IL6 (a), $\log _{10}$ TNF $\alpha$ (b), IGF1 (c), and total carbohydrate intake (d) with neutrophil phagocytic index. $r=$ Pearson's correlation coefficient.

Although little is known about the influence of exercise on the function of neutrophilic polymorphonuclear leukocytes in the geriatric populations [27], our results corroborate study that found lower phagocytic activity of neutrophils in sedentary elderly subjects compared to those physically active [28]. Study including middle-aged recreational soccer players showed higher phagocytic activity compared to sedentary subjects [29]. Although the cross-sectional nature of our study does not allow the establishment of causal relationship, these results suggest that the expected decline of the phagocytic response with aging $[3,5]$ may be at least partially reversed by adaptations induced by RT in populations under high risk of functional capacity decay.

On what concerns production of reactive oxygen species, our results did not show significant differences between groups, corroborating findings in different population strata and sportive modalities [29-31]. It has been well established that neutrophils may exhibit impaired capacity to produce superoxides in geriatric subjects $[32,33]$. We do not rule out that this result can, at least in part, be influenced by the expected age-related reduction of dehydroepiandrosterone (DHEA) to levels that may restrict potential benefits from physical exercise, since this circulating steroid has proven to be important to the synthesis of superoxides in neutrophils [34].

Regarding the fact that the monocyte functions have not been influenced by RT, it is likely that the monocytes/macrophages system may depend on its developmental state to benefit from endocrine mediators promoted by physical exercise [35]. Monocytes are a class of relatively immature circulating phagocytes that demand migration to tissues as a requirement for full cell specialization. It is plausible that different phagocytic indexes between monocytes and neutrophils arise from this relative immaturity of monocytic types or that the adaptations induced by our intervention have been insufficient to promote important endocrine changes to the point of favoring direct activation of monocytes. In line with this, we do not rule out the possibility that the sample size was insufficient to obtain a significant effect on monocytes' indexes.

The analysis of the inflammatory profile of the subjects in this study corroborates evidence from literature that associates increased IL6 levels with an age-dependent dysregulation of the innate immune system [34]. Regarding the circulating TNF $\alpha$ level, the results showed no significant associations with the phagocytic activity. This finding is in 
line with studies in human models that support the concept that TNF $\alpha$ is not a robust biomarker that reflects systemic inflammation in sedentary populations when compared to abnormal endocrine [36] and tissue [37] levels of IL6. Therefore, it is likely that the serum IL6 is a more robust predictor of physical condition in apparently healthy yet sarcopeniaprone geriatric populations, due to evidence that IL6 (but not $\mathrm{TNF} \alpha$ ) tends to be actively produced by the exercising muscle $[38,39]$.

Factors extrinsic to the immune system are implicated in the imbalance of the innate immune system with aging. Phagocytes are exposed to a variety of extraimmune agents (hormonal, mainly), which may affect their functional phenotype [7] by changes in intracellular signaling cascades [33], for instance. In our conditions, the phagocytic activity of neutrophils was not influenced by the circulating levels of insulin-like growth factor-1 (IGF1). Despite evidence that RT produces acute increases in serum IGF1 and in IGF1R on circulating monocytes [40], few studies have investigated the influence of RT on levels of this potent anabolic factor [4143], with inconsistent results [44]. It is likely that the different protocols analyzed and the small frequency of postexercise assessments could explain these results or that neuroendocrine (e.g., catecholamines) signals are more effective modulators of phagocytic functions than the GH axis [26].

Despite the fact that total energy and carbohydrate intakes produced significant impact on the phagocytic response of neutrophils, it is important to observe that statistical control for such covariable did not suppress the association found between $\mathrm{PhI}$ and the physical status (RT-adapted or sedentary) of the older women investigated. The women in the RT group exhibited lower total caloric intake compared to sedentary subjects probably due to the reduced carbohydrate intake. Even though the authors consider this result to be important since the amount of nutrients ingested has been associated with changes in both cellular and humoral responses $[10,45]$, no explanation can be provided at this point for such disparity in the pattern of energy consumption displayed by the subjects. But we suggest that calorie intake should be controlled in studies investigating the relationship between exercise/physical activity and phagocytic response.

The cross-sectional nature of this study is a limitation, so the results should be interpreted with care. Prevalence bias cannot be ruled out, since an uneven distribution of preexisting health conditions not considered in the setting up of the groups may have resulted in more elderly women with an exceptional immune status to be included in the RT-trained group, for instance. On their behalf, the authors sustain that their analyses were designed with the purpose of controlling potential confounding variables associated with the effects of acute infectious processes, use of immunomodulatory drugs, systemic levels of relevant immune mediators, and nutritional factors prior to the phagocytic and oxidative assays.

\section{Conclusion}

The main findings of this study suggest that circulating neutrophils (but not monocytes) in older women adapted to long-term RT program bear higher phagocytic capability in relation to those of sedentary counterparts. There was no influence of RT on the oxidative activity of either neutrophils or monocytes. We suggest that the nutritional parameters and circulating immunomodulators also correlate with the phagocytic index of neutrophils and therefore must be controlled in studies addressing the relationship between exercise/physical activity and the phagocytic response in populations susceptible to significant weaknesses.

\section{Disclosure}

The authors declare minor self-plagiarism by reusing elements from published work of their own to help them describe the sample and methods, providing appropriate reference.

\section{Conflict of Interests}

The authors declare that there is no conflict of interests regarding the publication of this paper.

\section{Authors' Contribution}

João Bartholomeu-Neto and Vinícius Carolino Sousa executed the phagocytic assays and laboratory assessments of the immune mediators. Aparecido Pimentel Ferreira and David Junger Fonseca Alves executed the clinical anthropometric assessments. Juliana Oliveira Toledo, Roberta Silva Paula, and Clayton Franco Moraes executed the pharmacological assessments, dietary registries, and medical component of the study, respectively. Ciro José Brito advised on the statistical analysis and interpretation of results. Otávio Toledo Nóbrega and Cláudio Córdova designed and coordinated the study and analyzed and interpreted the results. João BartholomeuNeto, Ciro José Brito, Roberta Silva Paula, Otávio Toledo Nóbrega, and Cláudio Córdova participated in the preparation of the original paper as well as the revision process.

\section{Acknowledgments}

This work was supported by FAPDF (Grant no. 193.000.0292012) and CNPq (Grant no. 471872/2011-3). Vinícius Carolino Sousa received a scholarship from CAPES, whereas Otávio Toledo Nóbrega received a fellowship for productivity in research from CNPq.

\section{References}

[1] B. J. Nicklas and T. E. Brinkley, "Exercise training as a treatment for chronic inflammation in the elderly," Exercise and Sport Sciences Reviews, vol. 37, no. 4, pp. 165-170, 2009.

[2] S. Giunta, "Is inflammaging an auto[innate]immunity subclinical syndrome?” Immunity \& Ageing, vol. 3, article 12, 2006.

[3] D. Aw, A. B. Silva, and D. B. Palmer, "Immunosenescence: emerging challenges for an ageing population," Immunology, vol. 120, no. 4, pp. 435-446, 2007.

[4] O. D. T. Nóbrega, V. P. Faleiros, and J. L. Telles, “Gerontology in the developing Brazil: achievements and challenges in public 
policies," Geriatrics and Gerontology International, vol. 9, no. 2, pp. 135-139, 2009.

[5] A. Panda, A. Arjona, E. Sapey et al., "Human innate immunosenescence: causes and consequences for immunity in old age," Trends in Immunology, vol. 30, no. 7, pp. 325-333, 2009.

[6] D. S. Senchina and M. L. Kohut, "Immunological outcomes of exercise in older adults," Clinical Interventions in Aging, vol. 2, no. 1, pp. 3-16, 2007.

[7] R. D. Stout and J. Suttles, "Immunosenescence and macrophage functional plasticity: dysregulation of macrophage function by age-associated microenvironmental changes," Immunological Reviews, vol. 205, no. 1, pp. 60-71, 2005.

[8] L. Álvarez-Rodríguez, M. López-Hoyos, P. Muñoz-Cacho, and V. M. Martínez-Taboada, "Aging is associated with circulating cytokine dysregulation," Cellular Immunology, vol. 273, no. 2, pp. 124-132, 2012.

[9] R. Solana, R. Tarazona, I. Gayoso, O. Lesur, G. Dupuis, and T. Fulop, "Innate immunosenescence: effect of aging on cells and receptors of the innate immune system in humans," Seminars in Immunology, vol. 24, no. 5, pp. 331-341, 2012.

[10] C. Córdova, F. Lopes-E-Silva Jr., A. S. Pires et al., "Longterm resistance training is associated with reduced circulating levels of IL-6, IFN-gamma and TNF-alpha in elderly women," NeuroImmunoModulation, vol. 18, no. 3, pp. 165-170, 2011.

[11] M. D. Phillips, M. G. Flynn, B. K. McFarlin, L. K. Stewart, and K. L. Timmerman, "Resistance training at eight-repetition maximum reduces the inflammatory milieu in elderly women," Medicine and Science in Sports and Exercise, vol. 42, no. 2, pp. 314-325, 2010.

[12] J. J. García, E. Bote, M. D. Hinchado, and E. Ortega, "A single session of intense exercise improves the inflammatory response in healthy sedentary women," Journal of Physiology and Biochemistry, vol. 67, no. 1, pp. 87-94, 2011.

[13] C. Córdova, V. C. Silva, C. F. Moraes, H. G. Simões, and O. T. Nóbrega, "Acute exercise performed close to the anaerobic threshold improves cognitive performance in elderly females," Brazilian Journal of Medical and Biological Research, vol. 42, no. 5, pp. 458-464, 2009.

[14] R. S. Paula, V. C. Souza, A. L. Benedet et al., "Dietary fat and apolipoprotein genotypes modulate plasma lipoprotein levels in Brazilian elderly women," Molecular and Cellular Biochemistry, vol. 337, no. 1-2, pp. 307-315, 2010.

[15] D. A. Lipschitz, "Screening for nutritional status in the elderly," Primary Care, vol. 21, no. 1, pp. 55-67, 1994.

[16] M. J. Brekke, M. L. Brekke, E. S. Quiter, J. R. Peters, R. M. Mullis, and D. B. Hunninghake, "Appropriate days for measuring intake of dietary fat and cholesterol," Annals of Nutrition and Metabolism, vol. 36, no. 5-6, pp. 318-327, 1992.

[17] P. M. Lührmann, B. M. Herbert, C. Gaster, and M. NeuhäuserBerthold, "Validation of a self-administered 3-day estimated dietary record for use in the elderly," European Journal of Nutrition, vol. 38, no. 5, pp. 235-240, 1999.

[18] S. T. Philippi, Tabela de composiçäo de alimentos: suporte para decisäo nutricional, Coronário, São Paulo, Brazil, 2002.

[19] W. J. Kraemer, A. Fry, N. Ratamess, and D. French, "Strength testing: development and evaluation of methodology," in Physiological Assessment of Human Fitness, P. Maud and C. Foster, Eds., pp. 115-138, Human Kinetics, Champaign, Ill, USA, 1995.

[20] D. Agin, D. Gallagher, J. Wang, S. B. Heymsfield, R. N. Pierson Jr., and D. P. Kotler, "Effects of whey protein and resistance exercise on body cell mass, muscle strength, and quality of life in women with HIV,' AIDS, vol. 15, no. 18, pp. 2431-2440, 2001.
[21] M. I. Muniz-Junqueira, L. M. F. Peçanha, V. L. da SilvaFilho, M. C. de Almeida Cardoso, and C. E. Tosta, "Novel microtechnique for assessment of postnatal maturation of the phagocytic function of neutrophils and monocytes," Clinical and Diagnostic Laboratory Immunology, vol. 10, no. 6, pp. 10961102, 2003.

[22] P. J. Lachmann and M. J. Hobart, "Complement genetics in relation to HLA," British Medical Bulletin, vol. 34, no. 3, pp. 247252, 1978.

[23] B. H. Park, S. M. Fikrig, and E. M. Smithwick, "Infection and nitroblue-tetrazolium reduction by neutrophils: a diagnostic acid," The Lancet, vol. 292, no. 7567, pp. 532-534, 1968.

[24] S. Mahbub, A. L. Brubaker, and E. J. Kovacs, "Aging of the innate immune system: an update," Current Immunology Reviews, vol. 7, no. 1, pp. 104-115, 2011.

[25] A. Desai, A. Grolleau-Julius, and R. Yung, "Leukocyte function in the aging immune system," Journal of Leukocyte Biology, vol. 87, no. 6, pp. 1001-1009, 2010.

[26] E. Ortega, E. Giraldo, M. D. Hinchado, L. Martín, J. J. García, and M. De La Fuente, "Neuroimmunomodulation during exercise: role of catecholamines as 'stress mediator' and/or 'danger signal' for the innate immune response," NeuroImmunoModulation, vol. 14, no. 3-4, pp. 206-212, 2007.

[27] R. J. Simpson, T. W. Lowder, G. Spielmann, A. B. Bigley, E. C. LaVoy, and H. Kunz, "Exercise and the aging immune system," Ageing Research Reviews, vol. 11, no. 3, pp. 404-420, 2012.

[28] H. Yan, A. Kuroiwa, H. Tanaka, M. Shindo, A. Kiyonaga, and A. Nagayama, "Effect of moderate exercise on immune senescence in men," European Journal of Applied Physiology, vol. 86, no. 2, pp. 105-111, 2001.

[29] S. Sasaki, T. Matsuura, R. Takahashi et al., "Effects of regular exercise on neutrophil functions, oxidative stress parameters and antibody responses against 4-hydroxy-2-nonenal adducts in middle aged humans," Exercise Immunology Review, vol. 19, pp. 60-71, 2013.

[30] V. Hack, G. Strobel, M. Weiss, and H. Weicker, "PMN cell counts and phagocytic activity of highly trained athletes depend on training period," Journal of Applied Physiology, vol. 77, no. 4, pp. 1731-1735, 1994.

[31] K. Kowatari, T. Umeda, T. Shimoyama, S. Nakaji, Y. Yamamoto, and K. Sugawara, "Exercise training and energy restriction decrease neutrophil phagocytic activity in judoists," Medicine and Science in Sports and Exercise, vol. 33, no. 4, pp. 519-524, 2001.

[32] D. Baylis, D. B. Bartlett, H. P. Patel, and H. C. Roberts, "Understanding how we age: insights into inflammaging," Longevity \& Healthspan, vol. 2, article 8, 2013.

[33] C. R. Gomez, V. Nomellini, D. E. Faunce, and E. J. Kovacs, "Innate immunity and aging," Experimental Gerontology, vol. 43, no. 8, pp. 718-728, 2008.

[34] A. C. Shaw, D. R. Goldstein, and R. R. Montgomery, "Agedependent dysregulation of innate immunity," Nature Reviews Immunology, vol. 13, no. 12, pp. 875-887, 2013.

[35] M. Gleeson and N. Bishop, "Special feature for the Olympics: effects of exercise on the immune system: modification of immune responses to exercise by carbohydrate, glutamine and anti-oxidant supplements," Immunology \& Cell Biology, vol. 78, no. 5, pp. 554-561, 2000.

[36] A. Bosutti, G. Malaponte, M. Zanetti et al., "Calorie restriction modulates inactivity-induced changes in the inflammatory markers C-reactive protein and pentraxin-3," Journal of Clinical 
Endocrinology and Metabolism, vol. 93, no. 8, pp. 3226-3229, 2008.

[37] M. J. Drummond, K. L. Timmerman, M. M. Markofski et al., "Short-term bed rest increases TLR4 and IL-6 expression in skeletal muscle of older adults," American Journal of Physiology-Regulatory Integrative and Comparative Physiology, vol. 305, no. 3, pp. R216-R223, 2013.

[38] N. Mathur and B. K. Pedersen, "Exercise as a mean to control low-grade systemic inflammation," Mediators of Inflammation, vol. 2008, Article ID 109502, 6 pages, 2008.

[39] A. M. W. Petersen and B. K. Pedersen, "The anti-inflammatory effect of exercise," Journal of Applied Physiology, vol. 98, no. 4, pp. 1154-1162, 2005.

[40] M. S. Fragala, A. R. Jajtner, J. R. Townsend et al., "Leukocyte IGF-1 receptor expression during muscle recovery," Medicine \& Science in Sports \& Exercise, vol. 47, no. 1, pp. 92-99, 2015.

[41] W. J. Kraemer, K. Häkkinen, R. U. Newton et al., "Effects of heavy-resistance training on hormonal response patterns in younger vs. older men," Journal of Applied Physiology, vol. 87, no. 3, pp. 982-992, 1999.

[42] S. Bermon, P. Ferrari, P. Bernard, S. Altare, and C. Dolisi, "Responses of total and free insulin-like growth factor-I and insulin-like growth factor binding protein-3 after resistance exercise and training in elderly subjects," Acta Physiologica Scandinavica, vol. 165, no. 1, pp. 51-56, 1999.

[43] R. G. de Souza Vale, R. D. de Oliveira, C. S. Pernambuco, Y. P. D. S. F. de Meneses, J. D. S. Novaes, and A. D. F. D. de Andrade, "Effects of muscle strength and aerobic training on basal serum levels of IGF-1 and cortisol in elderly women," Archives of Gerontology and Geriatrics, vol. 49, no. 3, pp. 343347, 2009.

[44] M. Orenstein and C. Friedenreich, "Review of physical activity and the IGF family," Journal of Physical Activity \& Health, vol. 1, no. 4, pp. 291-320, 2004.

[45] N. C. Bishop, A. K. Blannin, N. P. Walsh, P. J. Robson, and M. Gleeson, "Nutritional aspects of immunosuppression in athletes," Sports Medicine, vol. 28, no. 3, pp. 151-176, 1999. 


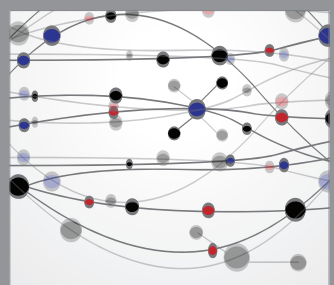

The Scientific World Journal
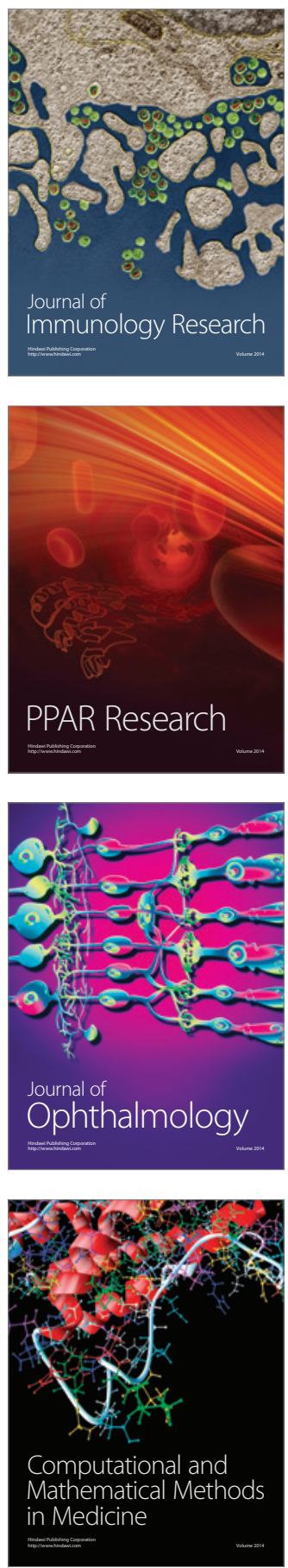

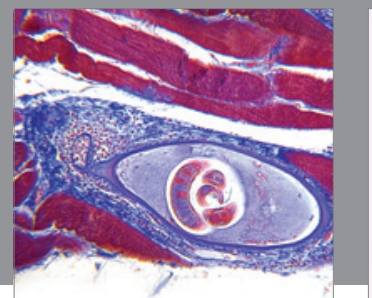

Gastroenterology

Research and Practice
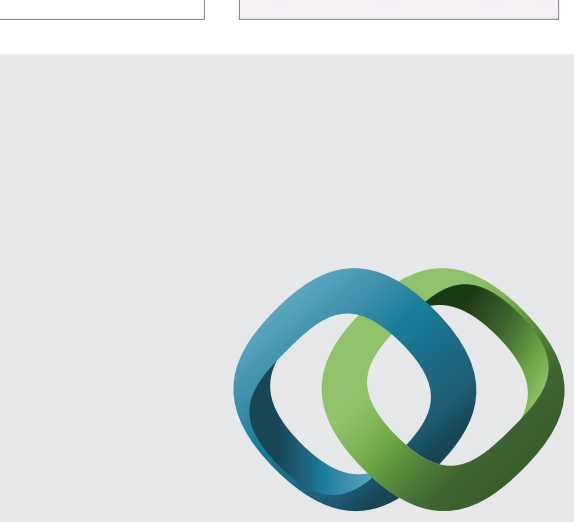

\section{Hindawi}

Submit your manuscripts at

http://www.hindawi.com
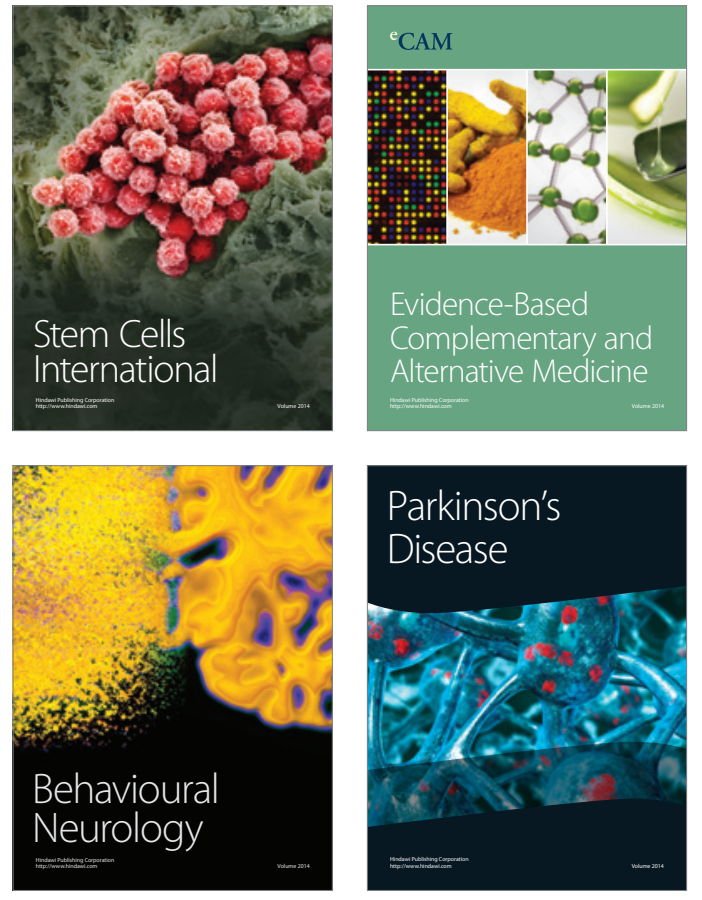
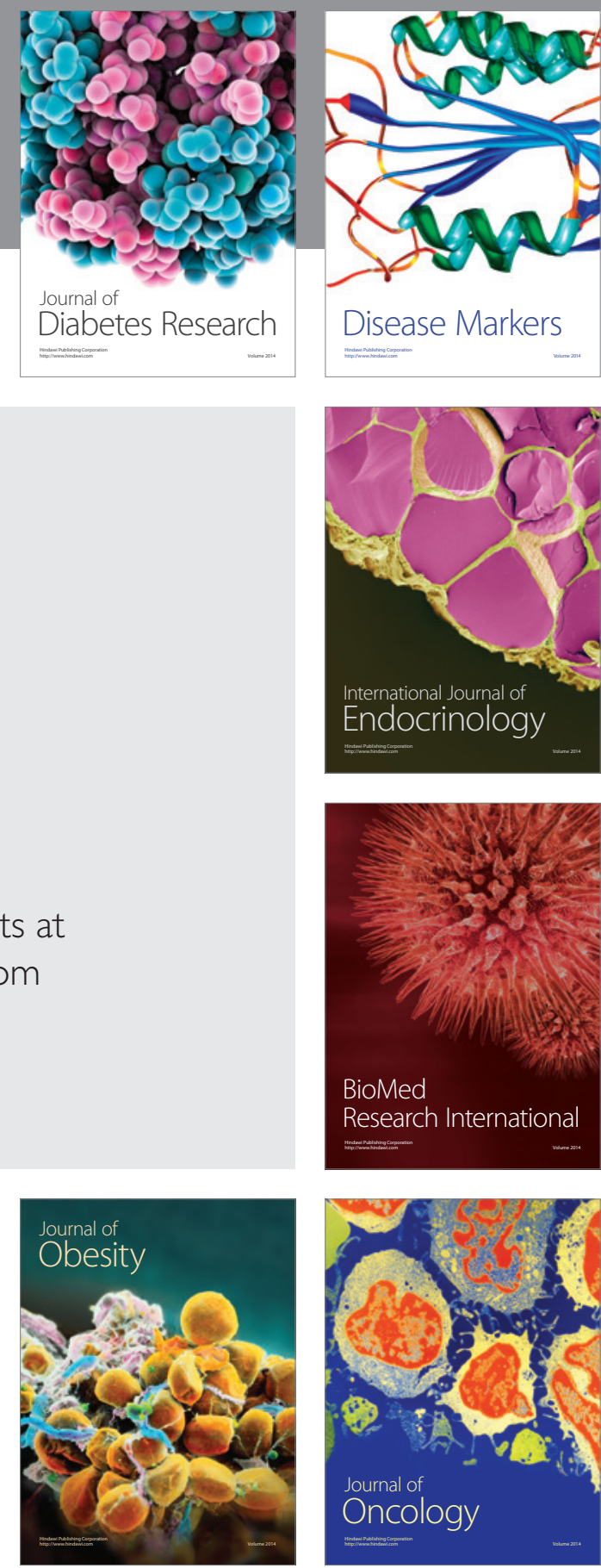

Disease Markers
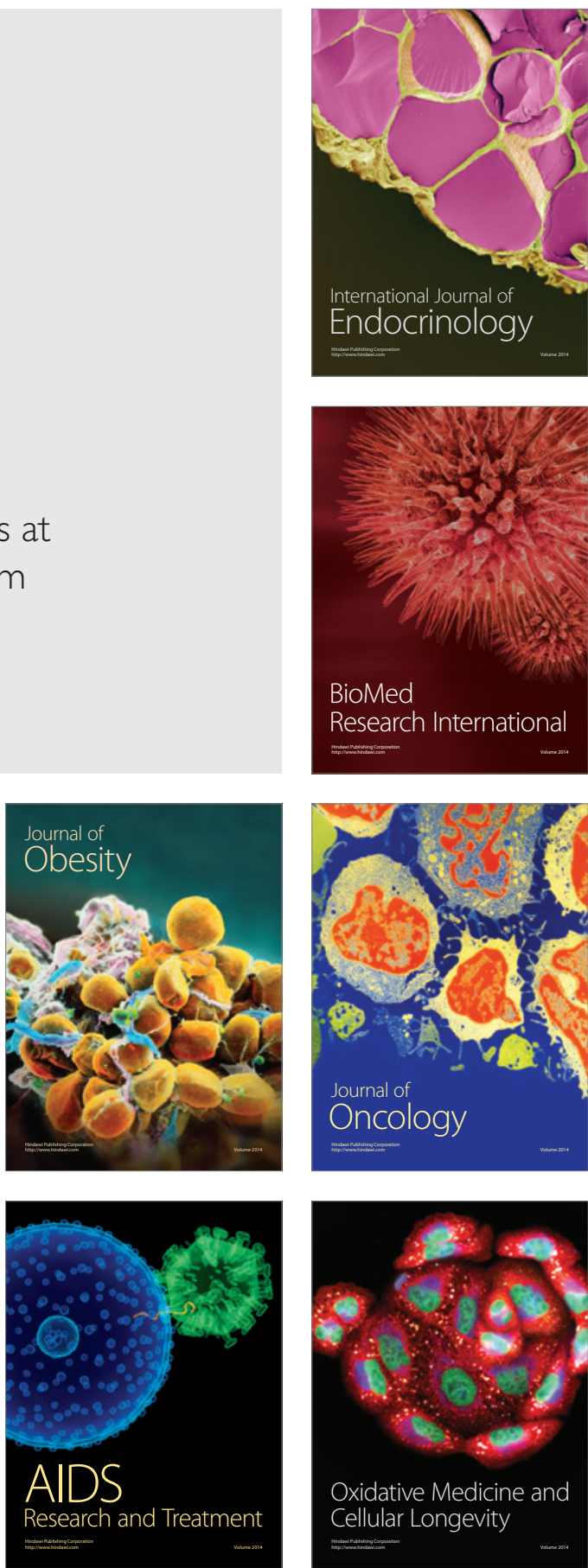